AperTO - Archivio Istituzionale Open Access dell'Università di Torino

\title{
Elastomechanical model of tumor invasion
}

\section{This is the author's manuscript}

Original Citation:

Availability:

This version is available http://hdl.handle.net/2318/103392

since

Published version:

DOI:10.1063/1.2398910

Terms of use:

Open Access

Anyone can freely access the full text of works made available as "Open Access". Works made available under a Creative Commons license can be used according to the terms and conditions of said license. Use of all other works requires consent of the right holder (author or publisher) if not exempted from copyright protection by the applicable law. 


\title{
Elastomechanical model of tumor invasion
}

\author{
Caterina Guiot \\ Department of Neuroscience, Università di Torino, Torino, Italy and CNISM, Torino, 10125 Italy
}

Nicola Pugno a)

Department of Structural Engineering and Geotechnics, Politecnico di Torino, Torino, 10129 Italy

Pier Paolo Delsanto

Department of Physics, Politecnico di Torino, Torino, 10010 Italy and Bioinformatics High Performance Computing Laboratories, Bioindustry Park of Canavese, Colleretto Giacosa, 10010 Italy

(Received 2 March 2006; accepted 19 October 2006; published online 4 December 2006)

\begin{abstract}
Tumor invasion concerns the tumor capability of colonizing the host by means of several complex biochemical processes. Since certain aspects of the problem present a striking resemblance with well known physical mechanisms, the authors propose here an analogy between tumoral invasive branching in a tissue and the mechanical insertion of a solid inclusion in an elastic material specimen. The model, which is an extension of a previous one, based on the universal growth law of West et al. [Nature (London) 413, 628 (2001)], is discussed in the case of multicellular tumor spheroids (and cords), but it may be adapted to understand invasion also in real tumors. (C) 2006 American Institute of Physics. [DOI: 10.1063/1.2398910]
\end{abstract}

The urgent need for a "system biology" approach to biomedical sciences, in particular oncology, has been widely recognized. ${ }^{1}$ It calls for a new theoretical framework of integrative cancer biology, bringing together the contributions of researchers from biology to mathematics, physics, information technology, and computational sciences. ${ }^{2}$

In the present contribution we wish to propose, as an example of cross-fertilization between different fields, ${ }^{3-5}$ a mechanistic description of tumor invasion as an extension of a previous cancer growth model ${ }^{5}$ based on the law of West $e t$ $a l .{ }^{3}$ The term "invasion" accounts for the capability of the tumor of colonizing its host by means of several biochemical processes, which can induce tissue degradation.

Our model is not intended as an alternative to previous models, but rather as being complementary to them, capturing some aspects of the invasion process, which present a striking resemblance with well known mechanisms of fracture mechanics. ${ }^{6}$ Incidentally, some concepts from fracture mechanics can account also for chemical diffusion. ${ }^{7}$ In fact, if we compare the growth of a tumor in a tissue with the physical insertion of a solid inclusion in an elastic fracturable material specimen, we find that from a purely mechanistic point of view the dynamics of the two processes is similar. To the pressure (or stress) exerted by the tumor (or invasion) on the host, the latter reacts with a compression and/or compaction of the former. ${ }^{8}$ As a consequence, the external stress is released allowing a new phase of tumor growth (invasion). The whole process shows the transition from the initial condition through a noninvasive growth to an invasive phase (when the matrix strength tolerance is reached), at the end of which the fracture-regrowth cycle (FRC) may start anew.

In the present contribution we limit for simplicity our investigation to the analysis of multicellular tumor spheroids (MTSs) and cords. In particular, MTSs are spherical aggregates of malignant cells, ${ }^{9,10}$ which can be grown in vitro under strictly controlled nutritional and mechanical conditions.

\footnotetext{
${ }^{\text {a)} E l e c t r o n i c ~ m a i l: ~ n i c o l a . p u g n o @ p o l i t o . i t ~}$
}

We call $R, M$, and $V$ the radius, mass, and volume of the MTS, $m$ and $v$ the average mass and volume of a single cell, and $N=M / m=V / v$ the total number of cells in the MTS. We use the indices $i$ and $f$ to denote the initial and final values, i.e., at the beginning and at the end of the FRC. We assume that $m$ is constant throughout the whole cycle, while $v$ changes, due to the external pressure $\sigma$, but with $v_{f}=v_{i}$, since we assume zero pressure at both ends of the FRC.

Let $B$ be the MTS input power from the surrounding matrix (a continuously replenished medium, such as agarose gel). Then, due to energy conservation, $B=\beta N+\eta d N / d t$, where the two terms on the right hand side represent the energy rate of consumption due to the metabolism and growth, respectively. $\beta$ is the metabolic rate for a single cell and $\eta$ the energy required to create a new cell. In Ref. 3, West et al. write $B=\alpha M^{p}$ with $p=3 / 4$ (see also Ref. 11).

In order to include the mechanistic effect of the interfacial pressure, we now add to the right hand side of the energy conservation law the mechanical term $\sigma d V / d t$. It follows that

$$
\eta \frac{d N}{d t}+\sigma \frac{d(v N)}{d t}-\gamma N^{p}+\beta N=0
$$

where $\gamma=\alpha m^{p}$. At any time during the FRC, $\sigma$ may be computed as the mechanical stress resulting from the compatibility between the elastic displacements in the MTS and in the annular region around it:

$$
\sigma=\frac{\sqrt[D]{N}-\sqrt[D]{N_{0}}}{c_{t} \sqrt[D]{N}+c_{m} \sqrt[D]{N_{0}}},
$$

where $D=3$ and $c_{m}$ and $c_{t}$ are the compliances of the matrix and tumor materials [given as functions of their elastic modulus and Poisson's ratio by Eqs. (7) and (9); for cylindrical symmetry, i.e., tumor cords, $c_{m}$ and $c_{t}$ are given by Eq. (14) and $D=2]$. Finally, to complete the system in the three unknowns $N, v$, and $\sigma$, we write the constitutive (stress-strain) equation: 


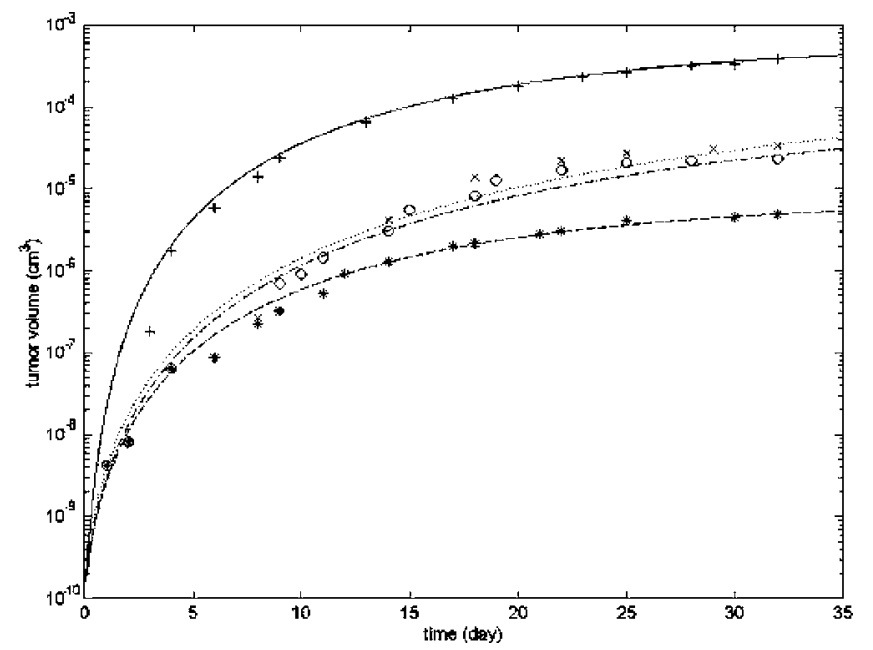

FIG. 1. Comparison between theoretical and observed time evolutions of the LS174T MTS volume. The theoretical lines were obtained using the parameter values listed in Table I (some of which resulted from a numerical fitting). They refer to MTS cultured in a gel with agarose concentrations of $0 \%(-), 0.5 \%(\cdots), 0.8 \%(---)$, and $0.9 \%(---)$. The corresponding experimental data (Ref. 13) are represented by the symbols,$+ \times, \bigcirc$, and ${ }^{*}$, respectively.

$$
\frac{d v}{d \sigma}=-3 c_{t} v
$$

Since $v$ cannot change too much during the FRC, it may be convenient to linearize Eq. (3) by replacing $v$ with $v_{0}$. The constant $3 c_{t}$ may be obtained using for a single cell the same procedure, leading to the same equation for the MTS [i.e., comparing the linearized equation (3) with Eq. (12)].

It may be interesting to note that the "first-order" asymptotic solution of Eq. (1) is identical to the one predicted by West et al., ${ }^{3}$ if the energy required to create a new cell $\eta$ is incremented by the mechanical factor $v_{0} \sigma$.

Returning to the mechanistic analogy, in which cracks correspond to the infiltration channels, failure is usually assumed to arise, for un-notched structures, when $\sigma$ reaches the material strength tolerance $\sigma_{C}$. In notched specimens, not the stress $\sigma$ but the stress-intensity factor $K$ must reach a critical value $K_{C}$ for fracture propagation; ${ }^{6}$ thus, $K \equiv \chi \sigma \sqrt{\pi l}=K_{C}$, i.e., the stress-intensity factor at the tip of a crack of length $l$ loaded by a stress $\sigma$ must be equal to the fracture toughness $K_{C}$ of the material; $\chi$ is a geometrical factor, e.g., for a crack at the edge of a large medium $\chi \approx 1.12$.

Recently a more powerful criterion (valid both for small and large values of $l$ ) for predicting the strength of solids has been derived, ${ }^{12}$ by simply removing the assumption of continuous crack propagation. Accordingly, the failure stress is estimated as

$$
\sigma_{f}=\frac{\sigma_{C}}{\sqrt{1+\left(\pi \chi^{2} \sigma_{C}^{2} / K_{I C}^{2}\right) l}},
$$

which represents an asymptotic matching between the two previously discussed solutions.

As a first example of application of our formalism, we now use Eqs. (1)-(3) to predict the time evolution of the tumor volume $V=N v$ for noninvasive MTS of human colon adenocarcinoma LS174T for different agarose concentrations, see Fig. 1. The parameters used for the calculations are Downloaded 05 Dec 2006 to 165.124.161.25. Redistribution subject
TABLE I. Values of the parameters in noninvasive (left column) and invasive (right column) cases and corresponding references. The parameters without reference are the result of a numerical fitting from experimental data. AC is the agarose concentration.

\begin{tabular}{ccc}
\hline \hline Parameter & LS174T MTS & U87MGmEGFR MTS \\
\hline $1 / c_{t}(\mathrm{kPa})$ & 3.8 & 2 \\
$1 / c_{m}(\mathrm{kPa})$ (unstressed) & 0 & $0.05^{\mathrm{a}}$ \\
$1 / c_{m}(\mathrm{kPa})$ at $0.5 \% \mathrm{AC}$ & $2.41^{\mathrm{b}}$ & $\ldots$ \\
$1 / c_{m}(\mathrm{kPa})$ at $0.8 \% \mathrm{AC}$ & $9.17^{\mathrm{b}}$ & $\ldots$ \\
$1 / c_{m}(\mathrm{kPa})$ at $0.9 \% \mathrm{AC}$ & $12.6^{\mathrm{b}}$ & $\ldots$ \\
$p$ & $\frac{3 \mathrm{c}}{4}$ & $\frac{3}{4}$ \\
$\gamma / \eta m_{c}^{1-p}\left(\mathrm{~g}^{0.25} /\right.$ day $)$ & 0.042 & 0.185 \\
$\beta / \eta\left(\right.$ day $\left.^{-1}\right)$ & 0.26 & 0.82 \\
\hline
\end{tabular}

${ }^{\mathrm{a}}$ Reference 14 .

${ }^{\mathrm{b}}$ Reference 13 .

${ }^{c}$ Reference 3 .

reported in the left column of Table I. The agreement with the corresponding experimental data ${ }^{13}$ is excellent.

Next, to treat the invasive phase, we apply Eq. (4). We analyze data from U87MGmEGFR glioma spheroids, ranging between 500 and $700 \mu \mathrm{m}$ in diameter, which were cultured for six consecutive days in a growth-factor-reduced Matrigel-OPTI-MEM medium mixture $(3: 1){ }^{14}$ In $40 \%$ of them there is an amplification of the epidermal growth factor receptor gene, producing mutant cells able to invade Matrigel. ${ }^{14}$ The parameter values are given in the right column of Table I. The observational data (empty dots in Fig. 2) correspond to an intermediate stage between growth in an unstressed medium (solid line) and in an elastic medium (dotted line), thus confirming the occurrence of invasion. The dashed line has been obtained by assuming that a critical stress occurs at a value $\sigma_{f}$ given by Eq. (4). Also in this case the agreement with the experimental data ${ }^{14}$ is very good.

Mathematical details. Let us consider a linear elastic isotropic spherical shell of inner and outer radii $a$ and $b$ subjected to inner and outer pressures $p$ and $q$, respectively. The

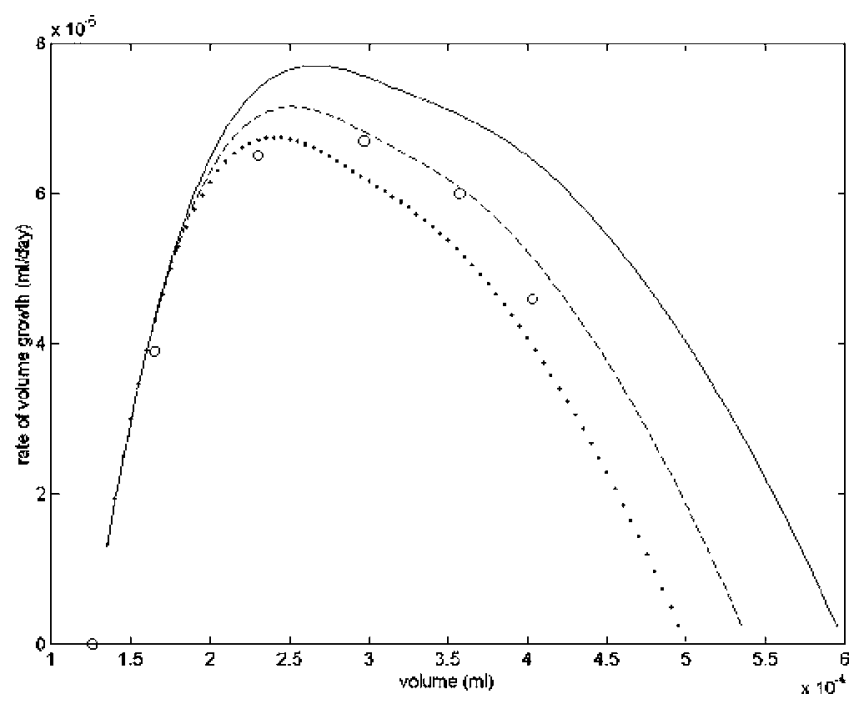

FIG. 2. Comparison between theoretical and observed rates of volume growth vs volumes of U87MGmEGFR glioma spheroids (Ref. 14). The empty dots represent the observational data and are fitted by the dashed line using the parameter values given in Table I. The solid and dotted lines correspond to the simulation results for growth in an unstressed and an elastic medium, respectively.

to AIP license or copyright, see http://apl.aip.org/apl/copyright.jsp 
radial and circumferential stress components at a generic radius $a<r<b$ are ${ }^{15}$

$$
\begin{aligned}
& \sigma_{r}=\frac{1}{b^{3}-a^{3}}\left(-b^{3} q+a^{3} p+\frac{a^{3} b^{3}}{r^{3}}(q-p)\right), \\
& \sigma_{\vartheta}=\frac{1}{b^{3}-a^{3}}\left(-b^{3} q+a^{3} p-\frac{a^{3} b^{3}}{2 r^{3}}(q-p)\right) .
\end{aligned}
$$

Linear isotropic elasticity and spherical symmetry yield the following for the radial component of the displacement $u$ and circumferential component of the strain $\varepsilon_{\vartheta}$ :

$$
u(r)=r \varepsilon_{\vartheta}=\frac{r}{E}\left((1-v) \sigma_{\vartheta}-\nu \sigma_{r}\right),
$$

where $E$ and $v$ are Young's modulus and Poisson's ratio of the material in the shell.

Let us now first apply Eqs. (5) and (6) for $a=R_{0}$ and $b$ $\rightarrow \infty$ (hence $q \rightarrow 0$ ). It follows for $r>R_{0}$, i.e., in the matrix surrounding the MTS, and $p=\sigma$,

$$
\begin{aligned}
& \sigma_{r}=-\frac{R_{0}^{3}}{r^{3}} \sigma, \\
& \sigma_{\vartheta}=-\frac{1}{2} \sigma_{r}, \\
& u(r)=c_{m} \frac{R_{0}^{3}}{r^{2}} \sigma, \\
& c_{m}=\left(1+v_{m}\right) /\left(2 E_{m}\right) .
\end{aligned}
$$

We use the indices $m$ and $t$ for the matrix and tumor, respectively. The radius of the deformed matrix (surrounding the MTS) at the pressure $\sigma$ is consequently

$$
A=R_{0}+u\left(R_{0}\right)=R_{0}\left(1+c_{m} \sigma\right) \text {. }
$$

Next we apply Eqs. (5) and (6) again, but with $a \rightarrow 0$, $b=R_{f}$, and $q=\sigma$. It follows that

$$
\begin{aligned}
& \sigma_{r}=\sigma_{\vartheta}=-\sigma, \\
& u\left(R_{f}\right)=-c_{t} \sigma R_{f}, \\
& c_{t}=\frac{1-2 v_{t}}{E_{t}} .
\end{aligned}
$$

Consequently the radius of the deformed MTS at the pressure $\sigma$, computed starting from the relaxed state at the end of the cycle, becomes

$$
B=R_{f}+u\left(R_{f}\right)=R_{f}\left(1-c_{t} \sigma\right) .
$$

The compatibility equation requires $A=B$ (contact at the interface between matrix and MTS), from which we derive

$$
\sigma=\frac{R_{f}-R_{0}}{c_{m} R_{0}+c_{t} R_{f}} .
$$

We now observe that the number of cells, $N$, increases, due to the term $\eta d N / d t$, only in the deformation between $R_{0}$ and $A$, and does not change sensibly during the quick relaxation from $B$ to $R_{f}$. Hence $N_{f}=N$. Also, since $R_{0}^{3}$ and $R_{f}^{3}$ are, respectively, proportional to $V_{0}=v_{0} N_{0}$ and $V_{f}=v_{f} N$, and $v_{0}$ $=v_{f}$, from Eq. (11) one obtains immediately Eq. (2).

For cylindrical symmetry (tumor channels) the extension of our formalism is straightforward. Eqs. (8)-(11) remain valid (but with different values of $c_{t, m}$ ); in fact Eqs. (5)-(7) become, respectively,

$$
\begin{aligned}
& \sigma_{r}=\frac{1}{b^{2}-a^{2}}\left(-b^{2} q+a^{2} p+\frac{a^{2} b^{2}}{r^{2}}(q-p)\right), \\
& \sigma_{\vartheta}=\frac{1}{b^{2}-a^{2}}\left(-b^{2} q+a^{2} p-\frac{a^{2} b^{2}}{r^{2}}(q-p)\right), \\
& u(r)=r \varepsilon_{\vartheta}=\frac{r}{E^{\prime}}\left(\sigma_{\vartheta}-v^{\prime} \sigma_{r}\right),
\end{aligned}
$$

with $E^{\prime}=E, \quad v^{\prime}=v$ for plane stress or $E^{\prime}=E / 1-v^{2}$, $v^{\prime}=v / 1-v$ for plane strain, and thus,

$$
\begin{aligned}
& \sigma_{r}=-\frac{R_{0}^{2}}{r^{2}} \sigma, \\
& \sigma_{\vartheta}=+\sigma_{r}, \\
& u=c_{m} \frac{R_{0}^{2}}{r} \sigma, \\
& c_{m}=\frac{1+v_{m}^{\prime}}{E_{m}^{\prime}}, \\
& c_{t}=\frac{1-v_{t}^{\prime}}{E_{t}^{\prime}} .
\end{aligned}
$$

This work has been partly supported by the Centre for the Development of a Virtual Tumor. Helpful discussions with T. S. Deisboeck and M. Griffa are gratefully acknowledged.

${ }^{1}$ NIH Guide to Integrative Cancer Biology Programs (2003).

${ }^{2}$ G. B. West and J. H. Brown, Phys. Today 57, 36 (2004).

${ }^{3}$ G. B. West, J. H. Brown, and B. J. Enquist, Nature (London) 413, 628 (2001).

${ }^{4}$ P. P. Delsanto, M. Griffa, C. A. Condat, S. Delsanto, and L. Morra, Phys. Rev. Lett. 94, 148105 (2005).

${ }^{5}$ C. Guiot, P. G. Degiorgis, P. P. Delsanto, P. Gabriele, and T. S. Deisboeck, J. Theor. Biol. 225, 147 (2003).

${ }^{6}$ A. A. Griffith, Philos. Trans. R. Soc. London, Ser. A 221, 163 (1920)

${ }^{7}$ D. J. Unger, W. W. Gerberich, and E. C. Aifantis, Scr. Metall. 16, 1059 (1982).

${ }^{8}$ T. S. Deisboeck, Y. Mansury, C. Guiot, P. G. Degiorgis, and P. P. Delsanto, Med. Hypotheses 65, 785 (2005).

${ }^{9}$ R. Chignola, A. Schenetti, G. Andrighetto, E. Chiesa, R. Foroni, S. Sartoris, G. Tridente, and D. Liberati, Cell Prolif 33, 219 (2000).

${ }^{10}$ M. T. Santini, G. Rainaldi, R. Romano, A. Ferrante, S. Clemente, A. Motta, and P. L. Indovina, FEBS Lett. 557, 148 (2004).

${ }^{11}$ A. Carpinteri and N. Pugno, Nat. Mater. 4, 421 (2005).

${ }^{12}$ N. Pugno and R. Ruoff, Philos. Mag. 84, 2829 (2004).

${ }^{13}$ G. Helminger, P. A. Netti, H. C. Lichtenbeld, R. J. Melder, and R. K. Jain, Nat. Biotechnol. 15, 778 (1997).

${ }^{14}$ V. D. Gordon, M. T. Valentine, M. L. Gardel, D. Andor-Ardo, S. Dennison, A. Bogdanov, D. A. Weitz, and T. S. Deisboeck, Exp. Cell Res. 289, 58 (2003).

${ }^{15}$ J. Prescott, Applied Elasticity (Dover, New York, 1946). 